\title{
Reasonable Energy-saving Scheduling Model of Large-scale Logistics Transport
}

\author{
Hao Huang \\ School of Economics and Management, Nanjing Polytechnic Institute, Nanjing,210048, China.
}

\begin{abstract}
. in the process of large-scale logistics and transport, the relationship between transport path selection of vehicles and transportation energy-saving is complex, this relationship is affected by the nonlinear application of vehicle transportation time, distance and cost. Reasonable energysaving scheduling method in traditional large-scale logistics and transport process requires the introduction of a large number of constraints, in order to fully establish the correlation between them, resulting in the modeling process of energy-saving scheduling in logistics transportation is too complex. Therefore, an optimization model of reasonable energy-saving scheduling in the process of large-scale logistics and transport based on grey logistics transportation model is proposed. By using the neural network method, the related data of transport goods are trained, so as to provide accurate data basis for the logistics route optimization. Based on the above data, the grey model of logistics and transport is established, and reasonable logistics and transport path is selected, so as to realize the logistics route optimization, and finally complete the reasonable energy-saving scheduling in the process of large-scale logistics and transport. The experimental results show that the improved algorithm for the path selection in the process of transportation and logistics, which can shorten the transportation time and transportation distance, and reduce the energy consumption in the process, so as to realize reasonable energy-saving scheduling in the process of large-scale logistics and transport.
\end{abstract}

Keywords: vehicle transportation; transport route; energy-saving scheduling.

\section{Introduction}

In the process of large-scale logistics and transport, the reasonable energy-saving scheduling of logistics transportation is a hot research topic in the field of logistics and transportation [1-3]. At present, the scheduling models mainly include the model based on ant colony algorithm, genetic algorithm and support vector machine algorithm [4-7]. Among them, the most commonly used is the scheduling model based on ant colony algorithm in the process of logistics and transportation. Due to the reasonable scheduling method of large-scale logistics and transport has a very broad prospects for development, it has become the focus of research [8-10].

However, traditional energy-saving scheduling methods of logistics transportation only consider the time, distance and cost of transport, ignoring the energy consumption in the process of transport vehicles, which is unable to meet the requirements of reasonable energy-saving scheduling of transportation.

In order to avoid the defects of traditional algorithm, an optimization model of reasonable energysaving scheduling in the process of large-scale logistics and transport based on grey logistics transportation model is proposed. Using the improved algorithm for the path selection in the process of transportation and logistics, which can shorten the transportation time and transportation distance, and reduce the energy consumption in the process, so as to realize reasonable energy-saving scheduling in the process of large-scale logistics and transport.

\section{Optimization Method of Reasonable Energy-saving Scheduling in Large Logistics Transportation Process}

\subsection{Related Data Training of Logistics}

By using the neural network method, the related data of transport object can be trained. The neurons in the hidden layer can be described by $k=\left(j k_{1}, j k_{2}, \cdots, j k_{r}\right)$, the output of the hidden layer 
is described by $j q=\left(j q_{1}, j q_{2}, \cdots, j q_{r}\right)$, the input data in output layer can be described by $a k=\left(a k_{1}, a k_{2}, \cdots, a k_{s}\right)$, the output results in output layer can be described by $a q=\left(a q_{1}, a q_{2}, \cdots, a q_{s}\right)$.

Use the following formula can calculate the parameters of the logistics route, to input to the neural network:

$$
\begin{gathered}
z(m-1)=\left[z_{1}(m-1), \cdots, m_{2}(m-1), z_{q+1}(m-1), \cdots, z_{q k}(m-1)\right]^{U} \\
=[a(m-1), \cdots, a(m-q), w(m-f), \cdots, w(m-f-p)]^{U} \\
z_{1}(m-1)=a(m-1), \cdots, z_{q}(m-1)=a(m-q), z_{q+1}(m-1)=w(m-f), \cdots, z_{q k}(m-1)=w(m-f-p) \\
q_{k}=q+p+1
\end{gathered}
$$

The output data of neural network can be calculated by using the following formula:

$$
\begin{gathered}
\hat{a}_{p}(m)=h(z(m-1) ; y)= \\
\phi\left[\sum_{l=1}^{Q_{2}}\left[y_{1 l_{1}} \phi\left[\sum_{k=1}^{Q_{1}} y_{k l} z_{k}(m-1)+\rho_{k l}\right]\right]+\rho_{2}\right]
\end{gathered}
$$

Among them, $k=1,2, \cdots, Q_{1}, l=1,2, \cdots, Q_{2}, Q_{1}$ and $Q_{2}$ are the number of the previous two neurons, $y_{k l}$ is the weight coefficient between the two layers, $\rho_{k l}$ is the offset.

According to the output of the neural network, it can get the following formula:

$$
\begin{aligned}
& \hat{z}_{p}(m+1)=h(z(m+n-1) ; y)= \\
& \phi\left[\sum_{l=1}^{Q_{2}}\left[y_{1 l_{1}} \phi\left[\sum_{k=1}^{Q_{1}} y_{k l} z_{k}(m+n-1)+\rho_{k l}\right]\right]+\rho_{2}\right]
\end{aligned}
$$

Among them, $n=1,2, \cdots, P, Q$ are the length of the frame of the output results in neural network. Supposing that the element number of input layer in neural network is $q$, the element number of hidden layer is $r$. Using the following formula can describe the logistics parameters which is needed to input to the neural network:

$$
Z=\left(z_{1}, z_{2}, \cdots, z_{q}\right)
$$

The optimal data set of logistics transportation network can be described by the following formula:

$$
e_{0}=\left(e_{1}, e_{2}, \cdots, e_{q}\right)
$$

\subsection{To Establish the Reasonable Energy-saving Scheduling Model of Grey Logistics Transport}

By using the reasonable energy-saving scheduling model of grey logistics transport can calculate the transport path in logistics lines, in order to obtain the most reasonable transportation mode in logistics transportation network, and realize the reasonable energy-saving scheduling in logistics transport process. The steps are as follows:

Setting that $I P(p, q)$ is the model function of reasonable energy-saving scheduling of grey logistics transport, under normal circumstances it will change constantly, which includes logistics variables and differential equation.

Setting that the set constituted of all logistics and transport destination is $z^{(0)}$, the results is made accumulation processing to acquire the new collection $z^{(0)}$. the acquired logistics and transport data need to satisfy the following conditions: 


$$
\frac{d z^{(1)}}{d v}+\chi z^{(1)}=w
$$

Among them, $\chi$ and $w$ are the unknown data in logistics transportation network. The above formula is transformed to obtain the following formula:

$$
z^{\wedge(1)}(k+1)=\left(z^{(1)}-\frac{w}{\chi}\right) e^{-\chi k}+\frac{w}{\chi}
$$

According to the least square method, to solve the problem of logistics network, it can get the following results:

$$
\hat{C}=\left[\begin{array}{l}
\chi \\
w
\end{array}\right]=\left(D^{U} D\right)^{-1} D^{U} A_{Q}
$$

Among them,

$$
\begin{aligned}
& D=\left[\begin{array}{cccc}
-\frac{1}{2}\left[z^{(1)}(1)+z^{(1)}(2)\right. & & 1 \\
-\frac{1}{2}\left[z^{(1)}(2)+z^{(1)}(3)\right. & & 1 \\
\cdots & \cdots & \cdots & \cdots \\
-\frac{1}{2}\left[z^{(1)}(p-1)+z^{(1)}(p)\right. & 1
\end{array}\right] \\
& A_{Q}=\left(z^{(0)}(2), z^{(0)}(3), \cdots, z^{(0)}(p)\right)^{U}
\end{aligned}
$$

The logistics data is introduced into differential equations can obtain reasonable energy-saving scheduling model of the gray logistics and transport described as follows:

$$
\hat{Z}^{(1)}(v+1)=\left(z^{(0)}(1)-\frac{w}{\chi}\right) e^{-\chi v}+\frac{w}{\chi}
$$

According to the method described above, it can establish the reasonable transportation scheduling model of grey logistics, so as to realize the reasonable scheduling in logistics and transport process.

\section{Experiment Results and Analysis}

In order to improve the effectiveness of reasonable scheduling optimization model in large-scale logistics and transport process based on grey logistics and transport model presented in this paper, it needs a test.

Using the traditional algorithm and the improved algorithm search transport route in the process of reasonable energy saving scheduling, the acquired vehicle running path can be represented by the following figures: 


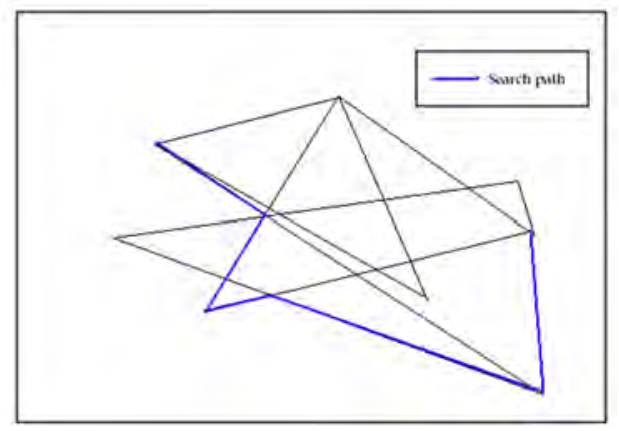

Figure 1. the reasonable energy-saving scheduling path by using the traditional algorithm to search

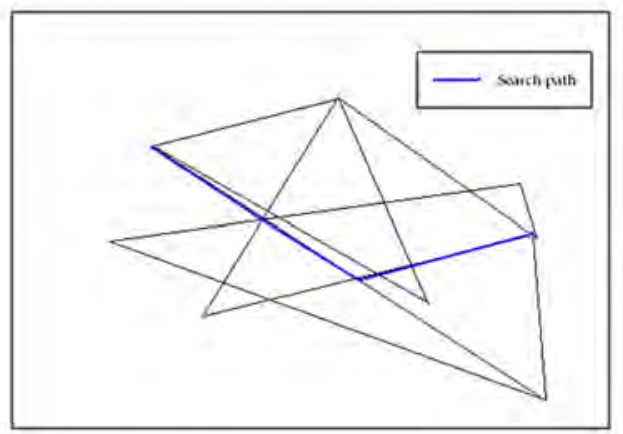

Figure 2. the reasonable energy-saving scheduling path by using the improved algorithm to search

From the comparison of the above figure, it can be seen that compared to the traditional algorithm, the reasonable energy saving scheduling path using improved algorithm can greatly shorten the running distance of vehicle transport.

The analysis of relevant data in the process of the experiment, can obtain the results as follows:

Table 1. experimental data of energy consumption in different algorithms

\begin{tabular}{ccc}
\hline $\begin{array}{c}\text { The number of } \\
\text { experiments }\end{array}$ & $\begin{array}{c}\text { The energy consumption of the } \\
\text { traditional algorithm (L) }\end{array}$ & $\begin{array}{c}\text { The energy consumption of the } \\
\text { improved algorithm (L) }\end{array}$ \\
\hline 1 & 102 & 88 \\
2 & 113 & 97 \\
3 & 98 & 88 \\
4 & 72 & 56 \\
5 & 101 & 94 \\
6 & 113 & 102 \\
7 & 67 & 55 \\
8 & 102 & 91 \\
9 & 97 & 88 \\
10 & 86 & 79 \\
\hline
\end{tabular}

The experiment show that the improved algorithm for the reasonable energy-saving scheduling in the process of large-scale logistics and transport, can effectively shorten the running distance and energy consumption, improve operational efficiency, to meet the transport requirements.

\section{Conclusion}

For the defects of that the reasonable energy-saving scheduling method in traditional large-scale logistics and transport process requires the introduction of a large number of constraints, in order to fully establish the correlation between them, otherwise it will result in the modeling process of energy-saving scheduling in logistics transportation is too complex. Therefore, an optimization model of reasonable energy-saving scheduling in the process of large-scale logistics and transport based on 
grey logistics transportation model is proposed. By using the neural network method, the related data of transport goods are trained, so as to provide accurate data basis for the logistics route optimization. Based on the above data, the grey model of logistics and transport is established, and reasonable logistics and transport path is selected, so as to realize the logistics route optimization, and finally complete the reasonable energy-saving scheduling in the process of large-scale logistics and transport. The experimental results show that the improved algorithm for the path selection in the process of transportation and logistics, which can shorten the transportation time and transportation distance, and reduce the energy consumption in the process, so as to realize reasonable energy-saving scheduling in the process of large-scale logistics and transport.

\section{References}

[1]. Cui Shiyue. Simulation of Logistics Vehicle Scheduling Model Considering Traffic Accident Interference Factors [J]. Computer simulation, 2014, 31 (4): 180-183.

[2]. Jia Shunping, Yin Xiangyong. Freight Delivery Process Based on VRPTW Model \& Rationalization Judgment [J]. Logistics technology, 2007, 26 (3): 36-39.

[3]. Wang Yong, Tang Haoyang, Qin Peng. A Logistical Discounted Model [J]. Chinese Journal of Chongqing University: Natural Science Edition, 2002, 25 (11): 118-121.

[4]. Jia Shunping, Peng Hongqin, Yin Xiangyong. Design in Network Logistics Information System Based on Vehicle Scheduling and Optimized Distribution [J]. Logistics technology, 2007, 26 (10): 111-113.

[5]. Deng Xue, Wang Xiaoping, Cao Liming. A LOGISTICS VEHICLE SCHEDULING OPTIMIZATION MODEL BASED ON REACHING PROBABILITY INFORMATION [J]. Computer applications and software, 2007, 24 (2): 14-15.

[6]. Wang Yueze. Study on transport rationalization of SINOTRANS INNER MONGOLIA COMPANY [J]. Journal of China Three Gorges University: Social Science Edition, 2011:64-66.

[7]. Cui Dong, Cui Hao. Study on the rationalization of logistics transportation under the condition of networked [J]. Economic and technological cooperation information, 2007, (21): 77-77.

[8]. Li Xin, Zhou Xiangzhen. A Catastrophe Operator Based on Logistics Lines Optimization Algorithm [J]. Bulletin of science and technology, 2012, 28 (12): 119-121.

[9]. Yu Dianxiang, Sun Qianchong, Dai Liandi. Study on Military Materials Delivery Process Based on VRPTW Model [J]. Logistics technology, 2009, 28 (10): 143-145.

[10]. Yang Shuanglin. Rationalization strategy analysis of logistics transportation based on distribution processing [J]. Heilongjiang science and technology information, 2008, (29): 88-88. 